\title{
Unimodality of the Betti numbers for Hamiltonian circle action with isolated fixed points
}

\author{
Yunhyung Cho And Min Kyu Kim
}

Let $(M, \omega)$ be an eight-dimensional closed symplectic manifold equipped with a Hamiltonian circle action with only isolated fixed points. In this article, we will show that the Betti numbers of $M$ are unimodal, i.e., $b_{0}(M) \leq b_{2}(M) \leq b_{4}(M)$.

\section{Introduction}

Let $(M, \omega, J)$ be a complex $n$-dimensional closed Kähler manifold. Then $(M, \omega, J)$ satisfies the hard Lefschetz property so that the Betti numbers are unimodal, i.e.,

$$
b_{i}(M) \leq b_{i+2}(M)
$$

for all $i \leq n-2$. In the symplectic case, the unimodality of the Betti numbers is not clear in general. Actually, there are many examples of closed symplectic manifolds where the hard Lefschetz property fails. But to the best of our knowledge, we could not find any example of a closed symplectic manifold where unimodality fails. In this paper, we will consider the following conjectural question raised by Susan Tolman, see [2].

Question 1.1. Let $(M, \omega)$ be a closed symplectic manifold with a Hamiltonian circle action. Assume that all fixed points are isolated. Then is the sequence of Betti numbers unimodal?

The reason why we put the condition "isolated fixed points" is that, as far as the authors know, all known examples of Hamiltonian circle actions with only isolated fixed points admit a Kähler structure. In particular, Karshon proved that every symplectic 4-manifold with a Hamiltonian circle action with only isolated fixed points admits an $S^{1}$-invariant Kähler structure, see [3]. In this paper, we will show 
Theorem 1.2. Let $(M, \omega)$ be an 8-dimensional closed symplectic manifold equipped with a Hamiltonian circle action with only isolated fixed points. Then the Betti numbers of $M$ are unimodal, i.e., $b_{0}(M) \leq b_{2}(M) \leq b_{4}(M)$.

We would like to give a remark that our method to approach this problem is purely "topological" in the sense that we do not use any geometric structure, like an almost complex structure or metric. Moreover, we cannot be sure whether our method does not work in higher-dimensional cases.

\section{Equivariant cohomology}

In this section, we briefly review an elementary equivariant cohomology theory and the localization theorem for a circle action which will be used in Section 3. Throughout this section, we will assume that every coefficient of any cohomology theory is $\mathbb{R}$. Let $S^{1}$ be the unit circle group and let $M$ be an $S^{1}$-manifold. Then the equivariant cohomology $H_{S^{1}}^{*}(M)$ is defined by

$$
H_{S^{1}}^{*}(M):=H^{*}\left(M \times_{S^{1}} E S^{1}\right),
$$

where $E S^{1}$ is a contractible space on which $S^{1}$ acts freely. Since $M \times_{S^{1}} E S^{1}$ has a natural $M$-bundle structure over the classifying space $B S^{1}:=E S^{1} / S^{1}$, the equivariant cohomology $H_{S^{1}}^{*}(M)$ admits an $H^{*}\left(B S^{1}\right)$-module structure. Note that $H^{*}\left(B S^{1} ; \mathbb{R}\right)$ is isomorphic to the polynomial ring $\mathbb{R}[u]$ where $u$ is of degree two. For the fixed point set $M^{S^{1}}$, the inclusion map $i: M^{S^{1}} \hookrightarrow M$ induces an $H^{*}\left(B S^{1}\right)$-algebra homomorphism

$$
i^{*}: H_{S^{1}}^{*}(M) \rightarrow H_{S^{1}}^{*}\left(M^{S^{1}}\right) \cong \bigoplus_{F \subset M^{S^{1}}} H^{*}(F) \otimes H^{*}\left(B S^{1}\right)
$$

and we call $i^{*}$ the restriction map to the fixed point set. Note that for any fixed component $F \in M^{S^{1}}$, the inclusion map $i_{F}: F \hookrightarrow M^{S^{1}}$ induces the natural projection $i_{F}^{*}: H_{S^{1}}^{*}\left(M^{S^{1}}\right) \rightarrow H_{S^{1}}^{*}(F) \cong H^{*}(F) \otimes H^{*}\left(B S^{1}\right)$. For every $\alpha \in H_{S^{1}}^{*}(M)$, we will denote by $\left.\alpha\right|_{F}$ the image $i_{F}^{*}\left(i^{*}(\alpha)\right)$. The main technique for proving Theorem 1.2 is the following, which is called AtiyahBott-Berlin-Vergne localization theorem.

Theorem 2.1 (A-B-B-V localization theorem). Let $M$ be a closed manifold with $S^{1}$-action with isolated fixed points. Let $\alpha \in H_{S^{1}}^{*}(M ; \mathbb{R})$. Then as an 
element of $\mathbb{R}(u)$, we have

$$
\int_{M} \alpha=\sum_{F \in M^{S^{1}}} \frac{\left.\alpha\right|_{F}}{e_{F}},
$$

where the sum is taken over all fixed points, and $e_{F}$ is the equivariant Euler class of the normal bundle to $F$.

Remark 2.2. Sometimes, the integral $\int_{M}$ is called an integration along the fiber $M$. If the given action is Hamiltonian, then $M$ is equivariantly formal so that we have $H_{S^{1}}^{*}(M) \cong H^{*}(M) \otimes H^{*}\left(B S^{1}\right)$ as an $H^{*}\left(B S^{1}\right)$-module, see [4]. Hence in the Cartan model, every equivariant cohomology class can be written as a sum of elements of the form $x \otimes u^{k} \in H_{S^{1}}^{*}(M) \cong H^{*}(M) \otimes$ $H^{*}\left(B S^{1}\right)$ and the operation $\int_{M}$ acts on the ordinary cohomology factor. Hence if $\alpha \in H_{S^{1}}^{*}(M)$ is of degree less than the dimension of $M$, then we have

$$
\int_{M} \alpha=\sum_{F \in M^{S^{1}}} \frac{\left.\alpha\right|_{F}}{e_{F}}=0 .
$$

When our manifold has a symplectic structure $\omega$ and the given action is Hamiltonian, then the equivariant cohomology satisfies a remarkable property as follows.

Theorem $2.3([4])$. Let $(M, \omega)$ be a closed symplectic manifold and $S^{1}$ acts on $(M, \omega)$ in a Hamiltonian fashion. Then the restriction map $i^{*}$ to the fixed point set is injective.

Theorem 2.3 enables us to study the ring structure of $H_{S^{1}}^{*}(M)$ more easily via the restriction map. For instance, assume that all fixed points are isolated. Then $H_{S^{1}}^{*}\left(M^{S^{1}}\right)$ is nothing but $\bigoplus_{F \in M^{S^{1}}} H^{*}\left(B S^{1}\right) \cong \bigoplus_{F \in M^{S^{1}}} \mathbb{R}[u]$. Hence we can think of an element $f \in H_{S^{1}}^{*}(M)$ as a function $i^{*}(f)$ from the fixed point set $M^{S^{1}}$ to the polynomial ring $\mathbb{R}[u]$ with one-variable $u$. Also, for any elements $f$ and $g$ of $H_{S^{1}}^{*}(M)$, the product $f \cdot g$ can be computed by studying $i^{*}(f \cdot g)$, which is simply the product of $i^{*}(f)$ and $i^{*}(g)$ on each fixed component.

Now, consider a Hamiltonian $S^{1}$-manifold $(M, \omega)$ with a moment map $H: M \rightarrow \mathbb{R}$. Then we may construct an equivariant symplectic class on $M \times S^{1} E S^{1}$ as follows. For the product space $M \times E S^{1}$, consider a two form $\omega_{H}:=\omega+d(H \cdot \theta)$, regarding $\omega$ as the pull-back of $\omega$ along the projection $M \times E S^{1} \rightarrow M$ and $\theta$ as the pull-back of a connection 1-form on the principal $S^{1}$-bundle $E S^{1} \rightarrow B S^{1}$ along the projection $M \times E S^{1} \rightarrow E S^{1}$. Here, 
we can think of $E S^{1} \rightarrow B S^{1}$ in terms of its finite-dimensional approximations. It is not hard to show that $\omega_{H}$ is $S^{1}$-invariant and vanishes on the fiber $S^{1}$ of $M \times E S^{1} \rightarrow M \times_{S^{1}} E S^{1}$. So we may push-forward $\omega_{H}$ to the Borel construction $M \times_{S^{1}} E S^{1}$ and denote by $\widetilde{\omega}_{H}$ the push-forward of $\omega_{H}$. Obviously, the restriction of $\widetilde{\omega}_{H}$ on each fiber $M$ is precisely $\omega$ and we call a class $\left[\widetilde{\omega}_{H}\right] \in H_{S^{1}}^{2}(M)$ an equivariant symplectic class with respect to $H$. By definition of $\widetilde{\omega}_{H}$, we have the following proposition.

Proposition $2.4([1])$. Let $F \in M^{S^{1}}$ be an isolated fixed point of the given Hamiltonian circle action. Then we have

$$
\left.\left[\widetilde{\omega}_{H}\right]\right|_{F}=H(F) u .
$$

\section{Main theorem}

Let $(M, \omega)$ be a closed symplectic manifold, $S^{1}$ be the unit circle group acting on $(M, \omega)$ in a Hamiltonian fashion, and $H: M \rightarrow \mathbb{R}$ be a moment map for the given action. Let $\nu_{F}$ be a normal bundle of $F$ in $M$. Then the negative normal bundle $\nu_{F}^{-}$of $F$ can be defined as the sub-bundle of $\nu_{F}$ whose fiber over $p \in F$ is the subspace of $T_{p} M$ tangent to an unstable submanifold of $M$ at $F$. We denote by $e_{F}^{-} \in H_{S^{1}}^{*}(F)$ the equivariant Euler class of $\nu_{F}^{-}$. McDuff and Tolman found a remarkable family of equivariant cohomology classes as follows.

Theorem 3.1 ([5]). Let $(M, \omega)$ be a closed symplectic manifold equipped with a Hamiltonian circle action with a moment map $H: M \rightarrow \mathbb{R}$. For each fixed component $F \subset M^{S^{1}}$, let $k_{F}$ be the index of $F$ with respect to $H$. Then given any cohomology class $Y \in H^{i}(F)$, there exists a unique class $\widetilde{Y} \in H_{S^{1}}^{i+k_{F}}(M)$ such that

1) $\left.\tilde{Y}\right|_{F^{\prime}}=0$ for every $F^{\prime} \in M^{S^{1}}$ with $H\left(F^{\prime}\right)<H(F)$,

2) $\left.\widetilde{Y}\right|_{F}=Y \cup e_{F}^{-}$,

3) the degree of $\left.\widetilde{Y}\right|_{F^{\prime}} \in H_{S^{1}}^{*}\left(F^{\prime}\right)$ is less than the index $k_{F^{\prime}}$ of $F^{\prime}$ for all fixed components $F^{\prime} \neq F$.

We call such a class $\widetilde{Y}$ the canonical class with respect to $Y$. In this case, when all fixed points are isolated, let $F$ be a fixed point of index $k_{F}$ and $1_{F} \in H^{0}(F)$ be the identity element of $H^{*}(F)$. Then Theorem 3.1 implies 
that there exists a unique class $\alpha_{F} \in H_{S^{1}}^{k_{F}}(M)$ such that

1) $\left.\alpha_{F}\right|_{F^{\prime}}=0$ for every $F^{\prime} \in M^{S^{1}}$ with $H\left(F^{\prime}\right)<H(F)$,

2) $\left.\alpha_{F}\right|_{F}=e_{F}^{-}=\prod w_{i}^{-} u$, where $\left\{w_{i}^{-}\right\}_{1 \leq i \leq \frac{k_{F}}{2}}$ are the negative weights of the $S^{1}$-representation on $T_{F} M$,

3) $\left.\alpha_{F}\right|_{F^{\prime}}=0$ for every $F^{\prime} \neq F \in M^{S^{1}}$ with $k_{F^{\prime}} \leq k_{F}$.

Now, we prove our main theorem.

Proof of Theorem 1.2. By the connectivity of $M$, the first inequality is obvious. Now, let us assume that $b_{2}(M)>b_{4}(M)$. Let $z_{1}, \ldots, z_{k}$ be the fixed points of index 2 and let $w_{1}, \ldots, w_{l}$ be the fixed points of index 4 where $k=b_{2}(M)$ and $l=b_{4}(M)$. Also, we denote by $\alpha_{i} \in H_{S^{1}}^{2}(M)$ the canonical class with respect to $z_{i}$ for each $i=1, \ldots, k$. Since we assumed that $b_{2}(M)=$ $k>l=b_{4}(M)$, the $k$ vectors $\left\{\left(\left.\alpha_{i}\right|_{w_{1}}, \ldots,\left.\alpha_{i}\right|_{w_{l}}\right)\right\}_{1 \leq i \leq k}$ are $\mathbb{R}$-linearly dependent. Hence there exists a non-zero class $\alpha=\sum_{i=1}^{k} c_{i} \alpha_{i}$ such that $\left.\alpha\right|_{w_{j}}=0$ for every $j$, so that $\alpha$ can survive only on the fixed points of index 2 , 6 or 8 . Now, consider an equivariant symplectic form $\widetilde{\omega}_{H}$ such that the maximum of $H$ is zero. If we let $\beta=\alpha^{2} \cdot\left[\widetilde{\omega}_{H}\right] \in H_{S^{1}}^{6}(M)$, then we have $\left.\beta\right|_{F}=\left(\left.\alpha\right|_{F}\right)^{2} \cdot H(F) u$ for each fixed point $F$ by Proposition 2.4. Since we took our moment map satisfying $\max H=0$, we have $H(F)<0$ for every fixed point $F$ which is not maximal so that all coefficients of $\left.\beta\right|_{F}$ are nonpositive. By Theorem $3.1(3)$, we have $\left.\beta\right|_{z_{i}}=\left(c_{i} e_{z_{i}}^{-}\right)^{2} \cdot H\left(z_{i}\right)$, and so $\left.\beta\right|_{z_{i}}$ is non-zero for every $i$ with $c_{i} \neq 0$. Hence $\beta$ is a non-zero class of degree 6 in $H_{S^{1}}^{*}(M)$ and survives only on the fixed points of index 2 or 6 . Applying the localization theorem to $\beta$, we have

$$
0=\int_{M} \beta=\sum_{F \in M^{S^{1}}} \frac{\left.\beta\right|_{F}}{e_{F}}=\sum_{\substack{F \in M^{1}, \\ \text { ind }(F)=2}} \frac{\left.\beta\right|_{F}}{e_{F}}+\sum_{\substack{F \in M^{1} \\ \text { ind }(F)=6}} \frac{\left.\beta\right|_{F}}{e_{F}}
$$

Since the coefficient of $u^{4}$ of $e_{F}$ is negative for every fixed point $F$ of index 2 or 6 , the right-hand side must be positive. Hence it is a contradiction.

\section{Acknowledgments}

The authors thank the anonymous referee for helpful suggestions. The second author is supported by GINUE research fund. 


\section{References}

[1] M. Audin, Torus actions on symplectic manifolds, Progress in Mathematics, 93, 2nd rev. ed., Birkhäuser Verlag, Basel, 2004.

[2] L. Jeffrey, T. Holm, Y. Karshon, E. Lerman and E. Meinrenkenf, Moment maps in various geometries, available at http://www. birs.ca/workshops/2005/05w5072/report05w5072.pdf.

[3] Y. Karshon, Periodic Hamiltonian flows on four-dimensional manifolds, Mem. Amer. Math. Soc. 141(672) (1999), 1-71.

[4] F.C. Kirwan, Cohomology of quotients in symplectic and algebraic geometry, Mathematical Notes, 31, Princeton University Press, Princeton, NJ, 1984.

[5] D. McDuff and S. Tolman, Topological properties of Hamiltonian circle actions, Int. Math. Res. Pap. 2006(2006), 1-77.

SCHOOL OF Mathematics

Korea Institute for Advanced Study

87 Hoegiro, Dongdaemun-gu, Seoul 130-722

REPUBLIC OF KOREA

E-mail address: yhcho@kias.re.kr

Department of Mathematics Education

Gyeongin National University of EducAtion

SAn 59-12, Gyesan-DOng, GyeyAnG-GU, InCHEON 407-753

REPUBLIC OF KOREA

E-mail address: mkkim@kias.re.kr

Received OCTOBer 23, 2013 